\title{
Eisen in Venezuela
}

Nach Berechnungen der UN Economic Commission for Europe (1) betrug 1970 der pro KopfVerbrauch an Stahl in Venezuela $155 \mathrm{~kg}$. Dies entspricht einerseits den niedrigsten Werten im Vergleich mit europäischen Ländern, ist aber anderseits der höchste Landeswert Südamerikas. In unserem Zusammenhang fällt vor allem ins Gewicht, dass Venezuela, obwohl es erst seit 1961 eine eisenschaffende Industrie besitzt, 1970 schon $57 \%$ seines Bedarfes aus eigener Produktion zu decken vermochte (ohne Berücksichtigung allfälliger Exporte).

Im Laufe der Sechzigerjahre verdoppelte sich ungefähr das Welttotal der Eisenproduktion, die Zuwachsrate Venezuelas lag dabei deutlich über dem Weltmittel. In Venezuela überstieg 1970 der Eisengehalt der geförderten Erze 14 Millionen $t$, während die Roheisenproduktion nur we nig mehr als eine halbe Million $t$ betrug. Fast 97\% der geförderten Erze wurden demnach exportiert.

Im Juli 1972 hatte der Verfasser Gelegenheit, die beiden für die skizzierte Entwicklung verantwortlichen Unternehmen zu besichtigen und an Ort und Stelle Informationen zu sammeln. In den Jahren 1969 - 1971 schwankten die durch die Orinoco Mining Company (OMC) exportierten Erzmengen zwischen 16 und 19 Millionen $t$, das sind mehr als drei Viertel der venezolanischen Eisenerzexporte. Ausbaupläne sehen schon für 197325 Millionen t vor. Wir wählten aus diesem Grunde die Orinoco Mining Company als Beispiel für einen Erzexporteur. Als Beispiel für eine eisenschaffende Industrie kam nur die Sidor (Siderurgica del Orinoco C. A.) in Frage. Beide Gesellschaften besitzen ihr Operationszentrum in Ciudad Guayana, einer modernen rasch wachsenden Stadt, welche an der Einmündung des Rio Caroni in den Orinoco in jenem Teil Venezuelas liegt, der als venezolanisch Guayana bezeichnet wird und an dessen Entwicklung die staatliche Corporacion Venezolana de Guayana (CVG) massgeblich beteiligt ist. Eine ausgezeichnete Dokumentation findet sich bei H. Tanner (2) und in den Berichten schweizerischer Journalisten, die ebenfalls 1972 auf Einladung der Regierung Venezuela bereisten (3).

Den Anstoss zur Entwicklung Venezuelas zu einem der wichtigsten Eisenproduzenten und -exporteure gaben nach dem Zweiten Weltkrieg die USA. Die US Steel Corporation wie die Bethle- hem Steel Corporation etablierten sich in $\mathrm{Ve}-$ nezuela. Orinoco Mining Company ist eine Tochtergesellschaft der US Steel und wurde 1949 gegründet. Der strukturelle Aufbau der Gesellschaft ist einfach: Erzabbau, Bahntransport zum Verladehafen, Sortieren und Mischen, Verladen und Transport nach überseeischen Absatzgebieten. Dabei werden etwa zwei Drittel von US Steel selbst, der Rest von anderen Gesellschaften übernommen. Wie in vielen anderen Eisenerzförderbezirken bilden auch hier die sogenannten "finos" (4) ein besonderes Problem. Sie fallen in starkem Masse bei den Umladeoperationen an und werden von der OMC nach einem neuen Verfahren briquettiert und ofengerecht gemacht. Dies ist, abgesehen von einigen Details, das Besondere bei der OMC.

Die meisten der riesigen Eisenerzvorkommen in venezolanisch Guayana sin sogenannte nationale Reserven. Die OMC baut innerhalb ihres Konzessionsgebietes am Cerro Bolivar, der sich rund $500 \mathrm{~m}$ über die Weite der Savanne erhebt, etwa $130 \mathrm{~km}$ südlich von Ciudad Guayana ein im Mittel 58-prozentiges Eisenerz ab. Am Fusse des Cerro Bolivar liegt die von der OMC gegründete Siedlung Ciudad Piar, eine sogenannte offene Stadt. Der ganze Cerro Bolivar besteht aus Eisenerz. Der Abbau erfolgt treppenweise mit Stufenhöhen von 15 Metern. Die Wände der Terrassen werden in grossen Massen abgesprengt und das Material durch mächtige Schlepper in die 90 Tonnen Ladegewicht aufweisenden Bahnwagen gebracht; am Fusse des Berges werden die je 125 Wagen zählenden Erzzüge zusammengestellt. Eine Ueberschlagsrechnung ergibt für 1970 bei regelmässiger Auslastung und kontinuierlicher Arbeit rund fünf Züge (ca. $50^{\prime} 000 \mathrm{t}$ ) im Tag. Obwohl die Bahnlinie nur einspurig ist, kann sie praktisch wie eine Doppelspurlinie befahren werden, weil lange Kreuzungsgeleise und zentralisierte Zugleitsysteme eine optimale Ausnützung gestatten.

Ciudad Guayana ist aus verschiedenen, teilweise alten Siedlungskernen zusammengewachsen. Eine dieser Zellen ist Puerto Ordaz, der am Orinoco liegende Verladehafen der OMC, zusammen mit den Verwaltungsgebäuden, Wohnsiedlungen etc. Hier endet die Erzbahn. Die schweren Bahnwagen werden einzeln durch

Prof. Dr. H. Boesch, Geogr. Inst. Universität Zürich, Blümlisalpstraße 10, 8006 Zürich 
Drehen in einer grossen Trommel entleert und rollen von dort selbständig in die Rangieranlagen, wo die Leerzüge zusammengestellt werden, während das Erz auf Förderbändern zu den Sortier-, Brech- und Mischanlagen gebracht wird. Die einzelnen Anlagen, einschliesslich der Verladeeinrichtungen auf die Erzdampfer, sind so aufeinander abgestimmt, dass in der Stunde $6000 \mathrm{t}$ Erz umgeschlagen werden können.

Mit der Verarbeitung der "finos" zu Erzbriketts befasst sich eine Tochtergesellschaft der OMC, Minerales Ordaz C. A. Das Werk steht unmittelbar neben den beschriebenen Verladeanlagen. An dieser Stelle soll auf das Besondere dieses neuen Brikettierverfahrens nicht eingegangen werden (5). Es genügt, darauf hinzuweisen, dass unter Zuhilfenahme von Erdgas aus den wenig weit entfernten ostvenezolanischen Feldern ofengerechte Erzbriketts mit einem $\mathrm{Fe}-\mathrm{Gehalt}$ von $86,5 \%$ erzeugt werden. Ein Teil wird exportiert, ein anderer Teil an die Sidor verkauft und in Guayana selbst verhüttet.

Die Beiträge der OMC an die venezolanische Volkswirtschaft können mit verschiedenen Massen gemessen werden. Eine Zusammenstellung der Gesellschaft zeigt, dass im Laufe von 18 Jahren (1954-1971) 4, 5 Milliarden Bolivar (ungefähr gleich viele Schweizer Franken) von der OMC der venezolanischen Volkswirtschaft zugeflossen sind. Dabei handelt es sich etwa zur Hälfte um Steuern. Verglichen mit der Erdölindustrie sind dies kleine Beiträge.

Nicht direkt in Geldeinheiten können andere und unseres Erachtens wichtigere Leistungen gemessen werden. Dazu gehört der Beitrag zur allgemeinen Entwicklung von Guayana und im besonderen zur Schiffbarmachung des Orinoco für 60'000 t (heute $80^{\prime} 000 \mathrm{t}$ ) Frachter bis Ciudad Guayana. OMC baute den $350 \mathrm{~km}$ langen Schifffahrtsweg aufgrund eines Vertrages mit der Landesregierung in der zweiten Hälfte der Fünfzigerjahre aus und hält ihn heute im Kontraktverhältnis mit dem Instituto Nacional de Canalizaciones vom Atlantik bis Puerto Ordaz offen (6).

Mit der allgemeinen Förderung der Entwicklung in venezolanisch Guayana betraut und mitverantwortlich für die Stadtplanung in Ciudad Guayana ist die staatliche Corporacion Venezolana de Guayana, CVG. Ciudad Guayana, als Stadt erst 1961 gegründet, besitzt eine rasch steigende Bevölkerungszahl und dürfte bald 200'000 erreichen; eine erste Ausbaugrösse liegt bei einer halben Million.

Westlich der Stadt, etwa $15 \mathrm{~km}$ vom Zentrum entfernt, wurde 1956 eine eisenschaffende Industrie begründet. 1964 übernahm die CVG über die Siderurgica del Orinoco C. A. (Sidor) die Anlagen und baute sie zu einem grossen integrierten Eisen- und Stahlwerk aus. Das Werk wird etappenweise vergrössert. Wenn wir die übliche Messstelle, d. h. die Ingot-produktion (Rohstahl) wählen, betrug die Kapazität 1970 $750^{\prime} 000 \mathrm{t}$ und 1971 (zweite Ausbauetappe) $1^{\prime} 000^{\prime} 000$ t. Dies entspricht einer Produktion von $750^{\prime} 000$ bis $800^{\prime} 000 \mathrm{t}$ für den Markt, weil 20-25 Prozent der Ingot-produktion innerhalb des Werkes als Zirkulationsschrott verbleiben. $\mathrm{Zu}$ den genannten Mengen kommen noch 50'000. bis $60^{\prime} 000 \mathrm{t}$ hinzu, welche als Roheisen die Giesserei erreichen. Nach Informationen der Sidor wurde das stark vereinfachte Diagramm gezeichnet; die Legende enthält alle notwendigen Angaben. Normalerweise wird eine installierte Kapazität aus den verschiedensten Gründen nicht voll ausgenützt. 1971 betrug das Gesamtgewicht der verschiedenen Produkte, welche das Werk verliessen, 580'000 t. Die Grösse eines Werkes kann verschieden beurteilt werden. Allgemein wird heute eine bedeutend grössere Kapazität als wirtschaftlich optimal betrachtet, beispielsweise 10 bis 15 Millionen $t$ bei den neusten japanischen Zentren. Andererseits stellten wir eingangs fest, dass theoretisch dieses Werk heute etwas mehr als die Hälfte des Landesbedarfes zu decken vermag.

Wirtschaftsgeographisch bietet Sidor vor allem zwei interessante Aspekte: Standortswahl und Technik der Erzverhüttung. Aus diesem Grunde dürfte Sidor auch im vergleichenden Unterricht Beachtung finden.

Die wichtigen Märkte für Stahl liegen in Venezuela entweder in den urbanen Zentren, das heisst vor allem in Caracas und Maracaibo, oder dort, wo die Erdölwirtschaft tätig ist. Eisenbahnen gibt es mit Ausnahme der Verbindung von Puerto Cabello nach Barquisimeto keine, dagegen braucht der Ausbau des Strassennetzes beträchtliche Stahlmengen. Weitere Verbrauchergruppen fallen nicht ins Gewicht. Mit Bezug auf diese Märkte liegt Sidor ausgesprochen peripher. Die Distanz nach Caracas auf dem Strassenweg beträgt $700 \mathrm{~km}$; dabei muss der Orinoco bei der Hauptstadt des Staates Bolivar auf 
Abb. 1: Cerro Bolivar

Gesamtansicht, Blickrichtung w in die etwa 500 m tiefer liegende Savanne, Juli 1972.
Abb. 2: Cerro Bolivar

Detail des treppenförmigen Abbaues des Erzkörpers (58\% Fe). Am linken Berghang noch sichtbar die ursprüngliche Oberfläche, Juli 1972.

Abb. 3: Sidor, Ciudad Guayana

Blick vom Verwaltungshochhaus gegen die Werkanlagen. Links (Rauchfahnen) die Roheisenhütte, rechts das Stahlwerk. Im Hintergrund der Orinoco, Juli 1972.
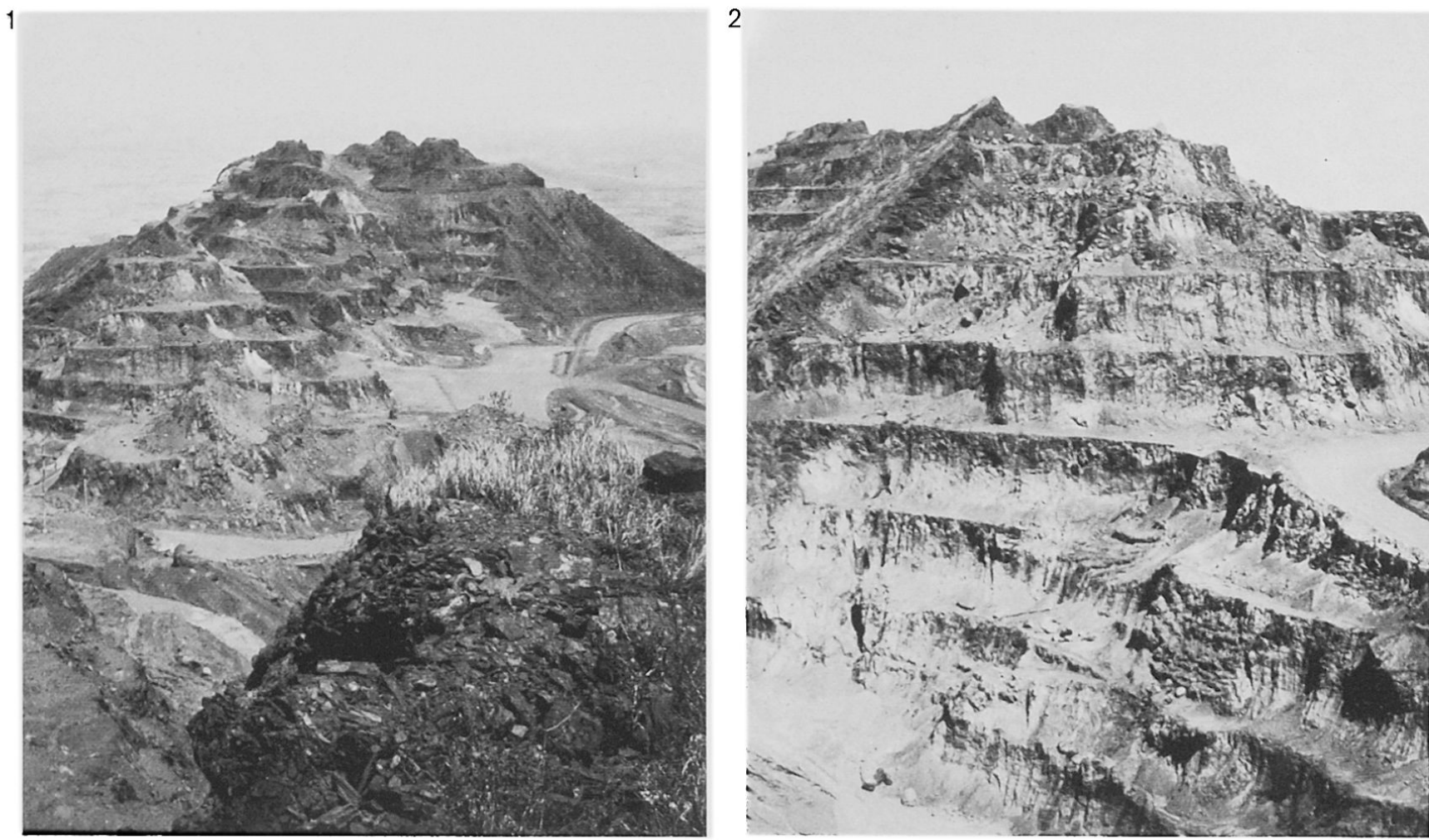

3

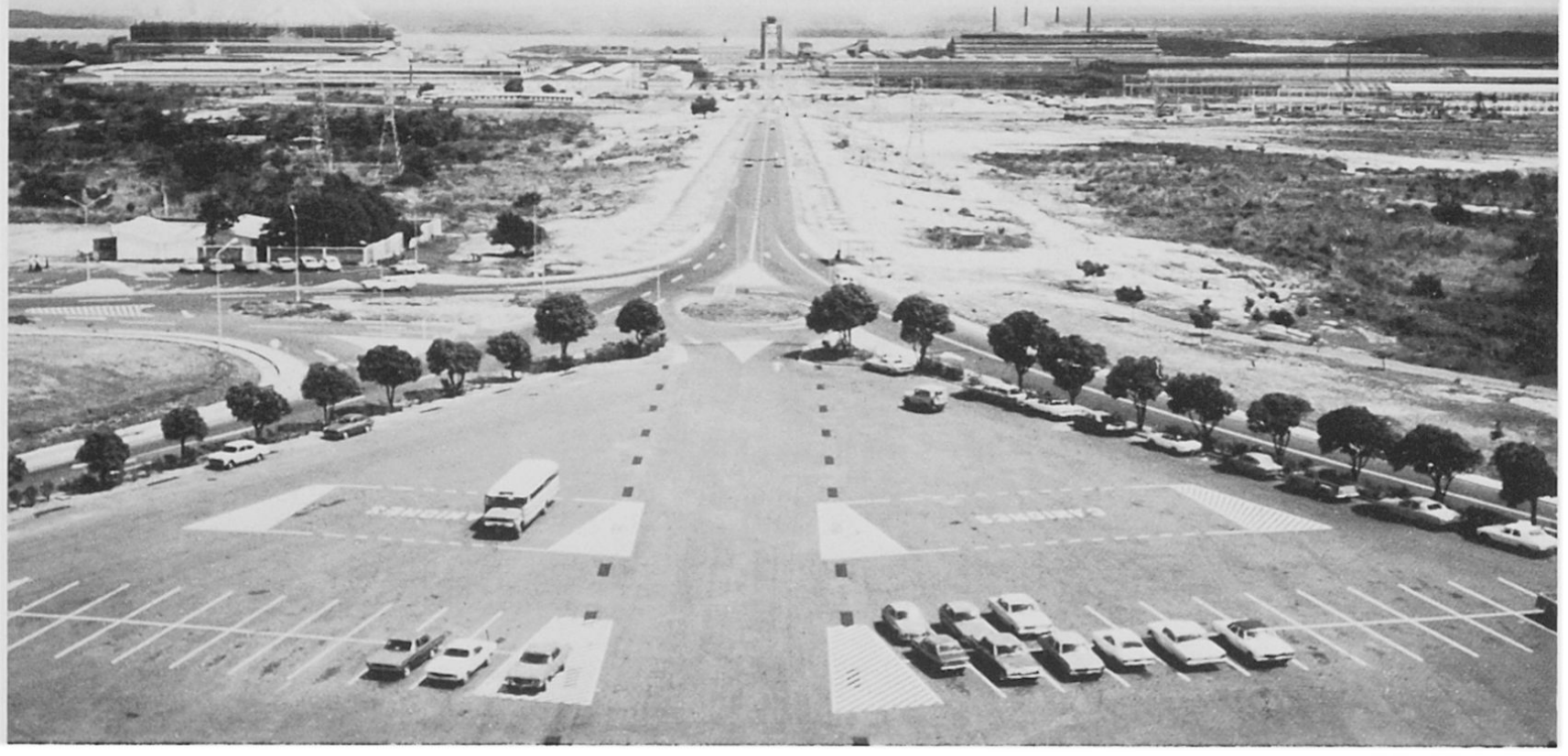


einer neuen Brücke gequert werden. Für schwere Fracht kommt nur der Wasserweg Orinoco abwärts und dann entlang der Küste in Frage.

Der gewählte Standort liegt auch mit Bezug auf die Schrottversorgung sehr ungünstig. Es erstaunt deshalb, dass im Stahlwerk das SiemensMartin Verfahren Anwendung findet. Fast aller Schrott ist werkinterner Zirkulationsschrott und nur kleine Mengen können von aussen zugekauft werden. Auch die Nähe der Erzbasis ist nicht entscheidend, weil es sich bei den Erzen Guayanas um hochwertige Eisenerze handelt, welche ohne weiteres lange Transporte ertragen.

Der entscheidende Grund für die Standortswahl ist, dass ausreichende und offenbar billige $\mathrm{Hy}-$ droelektrizität zur Verfügung steht. Unmittelbar vor seiner Einmündung in den Orinoco stürzt der Rio Caroni über eine ganze Treppe von prächtigen Kataraktstufen; andere Katarakte finden sich weiter flussaufwärts. An zwei Stellen sind - im Uebrigen mit einer bewundernswerten Schonung der Naturschönheiten - diese Wasserkräfte nutzbar gemacht worden. Die nördliche, untere Stufe wird in der Zentrale Macagua I, die obere bei Guri genutzt; bei Guri entstand durch Aufstauung des Rio Caroni ein grosser Stausee.

Die Anlagen der Sidor unterscheiden sich von andern Schwerindustriezentren durch das Fehlen des Koks-Hochofens. Das Erz und die Sinter werden in elektrischen Niederschachtöfen reduzierend erschmolzen. Dadurch wird der Koksbedarf, wie das Diagramm zeigt, weiter reduziert, weil Koks - der importiert werden muss - jetzt nur noch für den Reduktionsprozess benötigt wird; die Schmelzwärme liefert die Elektrizität (7).

In welchem Masse die Ausrichtung auf den nationalen Markt die Produktionsstruktur der Sidor beeinflusst, zeigt ihre Spezialisierung auf Stahlrohre. Wertmässig entfielen $197148 \%$ der Gesamtproduktion und $51 \%$ des im Lande selbst verkauften Anteils auf diese Gruppe. Die entsprechenden Mengenanteile liegen bei $26 \%$ und $30 \%$.

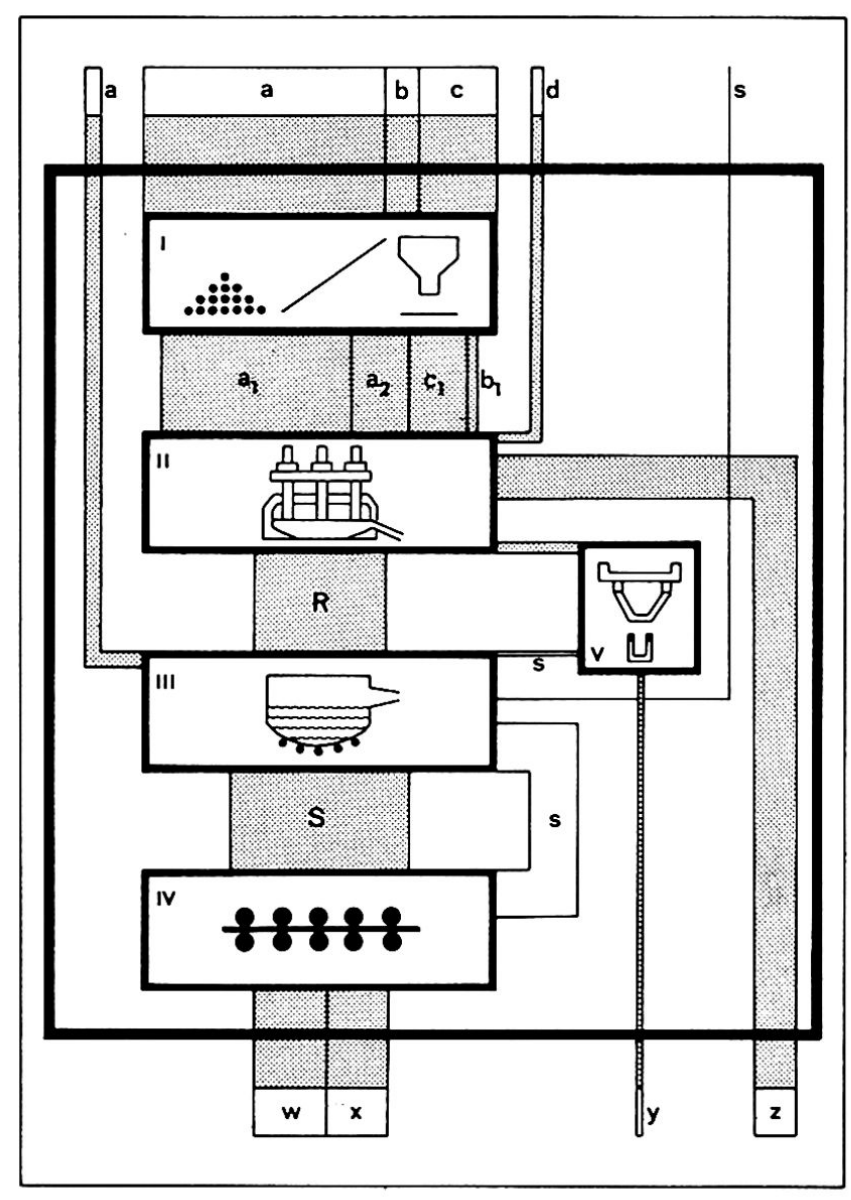

Fig. 1: Fließdiagramm Siderurgica del Orinoco

Das Diagramm wird von oben nach unten gelesen. Die in Klammern angegebenen Werte beziehen sich auf 1970/71 in '000 metr. Tonnen im Jahr. Zufuhr von Rohstoffen: a - Eisenerz (75+1020), b - Zuschlag (145), $c-$ Koks (324), d-Silica (53), s - zugekaufter Schrott (10). Innerhalb des stark ausgezogenen Gebietes liegen die Haupt-Werkeinheiten: I - Lagerung und Aufarbeitung, II - Elektroöfen, III - Stahlwerk, IV - Walzwerk etc., $V$ - Eisengießerei. $a_{1}-$ Sinter, $a_{2}-E r z, c_{1}-$ Koks, $b_{1}-$ Zuschlag. $\mathrm{R}$ - Roheisen (536 zum Stahlwerk und 53 in die Gießerei). S - Rohstahl zum Walzwerk etc. (750).

Die im Werk rückläufigen Schrottmengen (Zirkulationsschrott) sind als weiße Bänder dargestellt; s - von I zu III (200) und von V zu III (20).

Das Werk verlassen: $w$ - Stahlrohre (295), $x$ - andere Produkte des Walzwerkes etc. (255), y - Erzeugnisse der Gießerei (30), z - Schlacken granuliert (185).

Die Zahlen, welche für die Konstruktion des Diagrammes verwendet werden mußten, sind wie jene im Text verschiedenen Dokumentationen der Sidor entnommen worden; sie zeigen teilweise beträchtliche Unterschiede. 


\section{$\underline{\text { Notizen }}$}

(1) UN Statistical Yearbook 1971, New York 1972.

(2) Tanner H. : Venezolanisch Guayana - Planung und Entwicklung einer südamerikanischen Industrielandschaft, Plan 6/26, 1969.

(3) beispielsweise in Neue Zürcher Zeitung (Heini E.) nos. 386, 388 und 389,1972 , oder Tagesanzeiger (Berger R.) am 30.8. , 1.9. und 4.9. 1972 .

(4) Vergleichszahlen brasilianischer Erze finden sich bei Boesch H. : Der Eisenberg von Itabira und das Hüttenwerk Monlevade (Mi- nas Gerais, Brasilien), Z. f. Wirtsch. geographie $2 / 3,1959$, p. 36 .

(5) beschrieben bei Tanner H. (1969) und NZZ no. 389 (1972).

(6) OMC: Orinoco Mining Company-Its History and Development, 1972 und zahlreiche Einzeldokumentation.

(7) C. V. G. Siderurgica del Orinoco C. A. , Dokumentation 1972.

\section{Literaturbesprechung}

DONNER W. : Nepal - Raum, Mensch und Wirtschaft, Bd. 32 der Schriften des Instituts für Asienkunde in Hamburg, Otto Harrassowitz, Wiesbaden 1972, 506 Seiten, 122 Kartenskizzen, 32 Schaubilder, mehrfarbige Faltkarte, Preis: DM 128. -

Im Vorwort zu seinem Werk stellt der Verfasser sein Anliegen mit den Worten vor: "Das Buch will Nepal unter einem ganz bestimmten Aspekt darstellen. Wir gehen dabei von der Ueberzeugung aus, dass die Beziehungen zwischen Landschaft und Wirtschaft, die bekanntermassen überall bestehen, im Falle Nepals besonders stark zum Ausdruck kommen und besonders gravierend für die Entwicklungspolitik sind. Es dürfte nur wenige Lärider auf der Erde geben, wo die Natur auf kleinem Raum dem Entwicklungsprozess eine solche Fülle von Widerständen der verschiedensten Art entgegensetzt....". Diese Abhängigkeit und Wechselbeziehung zwischen "Raum, Mensch und Wirtschaft" liegt fast jedem Kapitel der vorliegenden umfangreichen Arbeit zugrunde. Dem Leser wird klar und deutlich die Notsituation Nepals vor Augen geführt, ihre vielfältigen Ursachen aufgezeigt und auf die im jetzigen Zeitpunkt oft hoffnungslos scheinende Aussicht auf eine baldige spürbare Besserung der Lage hingewiesen. Die Ergebnisse aus Untersuchungen, welche der Autor zum grossen Teil selbst durchgeführt hat, zeigen, zusammen mit dem spärlich vorhandenen statistischen Material, ein klares, wenn auch in vielen Belangen unvollständiges Bild der jetzigen wirtschaftlichen und sozialen Lage. Dass der Autor diese Unvollständigkeit und wahrscheinliche Ungenauigkeit vieler Daten deutlich herausstellt, und nicht versucht, aus spärlichen und ungenügenden Angaben Schlüsse zu ziehen, berührt sehr angenehm. Wer erwartet, dass Donner für die mannigfaltigen Probleme Nepals eine Patentlösung bereithält, wird enttäuscht. Es werden vielmehr mögliche Lösungswege vorgeschlagen, aber nicht, ohne dass auf die Probleme, welche diese nach sich ziehen, hingewiesen wird. Dies wird vor allem deutlich in den Kapiteln über Verkehrs-, Hydro-, Agrar-, Industrie- und Handelsgeographie, Böden und Bodenverwüstung, Bewässerungs - und Forstwirtschaft. Diese Kapitel bilden zusammen mit einer ausführlichen Sozialgeographie, einer Beschreibung der $\mathrm{Kli}-$ ma- und Vegetationszonen und einer physischen Geographie und räumlichen Gliederung den ersten und Kernteil des Werkes unter dem Untertitel "Nepal als Lebens - und Wirtschaftsraum". Die Einführung beginnt mit einer globalgeographischen Orientierung; ihr folgt ein kurzer geschichtlicher Abriss, aufgebaut auf die spärlichen Ueberlieferungen, die sich fast ausschliesslich auf das Kathmandutal beschränken und deutlich machen, weshalb Kathmandu heute noch den Staat Nepal schlechthin verkörpert, ob- 